\title{
Esters from carbofunctionalized organostannanes via Stille coupling ${ }^{1}$
}

\author{
John A. Soderquist, $*$ Izander Rosado, ${ }^{2}$ Yernan Marrero, ${ }^{3}$ and Carlos Burgos ${ }^{4}$ \\ Department of Chemistry, University of Puerto Rico San Juan, PR 00931-3346 \\ E-mail: jas@janice.uprr.pr
}

Dedicated to Prof. Alfred Hassner on the occasion of his $70^{\text {th }}$ birthday

(received 28 Feb 01; accepted 20 Sep 01; published on the web 28 Sep 01)

\begin{abstract}
The palladium-catalyzed cross coupling of organostannanes with chloroformates has been expanded to provide an improved route to unsaturated esters (63-85\%), including ?-methoxylated derivatives, through a mild one-pot procedure.
\end{abstract}

Keywords: Organostannanes, unsaturated esters, palladium-catalyzed cross coupling, Stille coupling

\section{Introduction}

Organostannanes provide the synthetic chemist with a wide variety of versatile reagents for both functional group conversions and carboskeletal construction ${ }^{-5}$ Stable enough to be isolable in pure form, they are sufficiently reactive to undergo a variety of highly selective transformations. Moreover, they are available from a range of organometallic procedures as well as from hydrostannylation. ${ }^{6}$ In addition to their well-known transmetallation and free-radical chemistry, the pioneering work of the late John K. Stille demonstrated their amazing versatility in the Pdcatalyzed cross coupling of these organometallics with electrophilic carbon substrates (Stille Coupling). ${ }^{5}$ Spawning numerous subsequent studies, sustained interest in this coupling process has resulted, not only in better procedures, ${ }^{5 \mathrm{~d}, 7}$ but also, in its providing the key step in the synthesis of numerous important natural products and useful intermediates. ${ }^{8}$

Over two decades ago, Hassner and Soderquist described the preparation of $\alpha$ ?methoxyvinyltin compounds and related metalloidal derivatives. ${ }^{6 \mathrm{~b}}$ These functionalized organometallics later proved to be remarkably efficient partners in the Stille protocol providing, for example, $\alpha$-methoxyenones and, subsequently, $\alpha$-diketones from acid chlorides and $(\alpha$ methoxyvinyl)trimethyltin. ${ }^{9}$ Since that time others have since utilized this coupling with chloroformates and carbamoyl chlorides for the preparation of esters and amides, respectively, 
from vinyl, aryl and heterocyclic organotin derivatives. ${ }^{10}$ While acceptable yields (ca. 70\%) have been generally obtained through this process, the conditions employed are generally harsh and the examples described include only a single example of this coupling with ( $\alpha$ alkoxyvinyl)tins. ${ }^{10 b}$ We felt that because such functionalized organotins are normally isolable, stable compounds which are available from numerous methods, the ( $\alpha$-alkoxyvinyl)tins and related compounds deserved more study.

\section{Results and Discussion}

Representative functionalized stannanes were selected ${ }^{5,11}$ which would extend the method to include the preparation of pyruvate ester derivatives and stereodefined synthetic intermediates. These were coupled to either ethyl or menthyl chloroformate using the original Stille conditions with neither $\mathrm{AsPh}_{3}$ nor $\mathrm{CuI}$ additives required. Efficient coupling was obtained in all of the examples examined (Table 1). The conditions $\left(\mathrm{BnPdCl}^{2} \mathrm{PPh}_{3}\right]_{2}(1-2 \%) ; \mathrm{PhH}$, reflux) which had been previously employed for the acid chloride couplings ${ }^{9}$ also proved to be quite successful for these substrates ( $c f$. 3a, $85 \%$ vs PhMe/HMPA: $\left.\left.\left(\mathrm{R}^{\prime \prime}=i-\operatorname{Pr}\right), 66 \%\right)\right)^{10 a}$

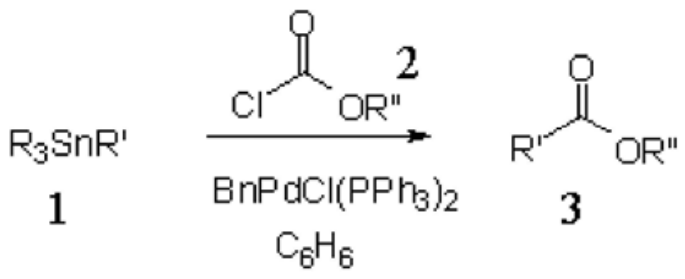

The protected pyruvates (3c,d) were prepared both as the ethyl and (-)-menthyl derivatives. Simple trans-vinylstannanes exhibited no significant stereochemical drift, with the isomerically pure 1f producing the pure trans- $\beta$-silylacrylate ester 3g. However, with the (Z)-1-methoxy-1,3butadienylstannane 1d, partial loss of stereochemistry occurs $(3 \mathbf{e} E / Z=70: 30)$ in the coupling process, a common phenomenon for less thermodynamically stable dienes [MMX calculations suggest that either Z-diene or E-3e isomerization is energetically favorable]., ${ }^{5,12}$ Despite this limitation, the Stille protocol is a highly effective tool for the carboalkoxylation of organostannanes which has been further demonstrated in the present study. Of particular significance is the fact that it has been shown for the first time that $\alpha$-methoxylated unsaturated esters can be efficiently assembled from $\alpha$-methoxyvinylstannanes and chloroformates through the Stille protocol. 
Table 1. Esters from organostannanes and chloroformates via Stille coupling

\begin{tabular}{ccccccc}
\hline $\mathbf{1}$ & $\mathrm{R}$ & $\mathrm{R}^{\prime}$ & $\mathbf{2}$ & $\mathrm{R} "$ & $\mathbf{3}$ & ${\text { Yield }(\%)^{\mathrm{a}}}^{\mathrm{a}}$ \\
\hline $\mathbf{a}$ & $n-\mathrm{Bu}$ & $\mathrm{Ph}$ & $\mathbf{a}$ & $\mathrm{Et}$ & $\mathbf{a}$ & 85 \\
$\mathbf{b}$ & $\mathrm{Me}$ & $n-\mathrm{C}_{5} \mathrm{H}_{11} \mathrm{C} \equiv \mathrm{C}$ & $\mathbf{a}$ & $\mathrm{Et}$ & $\mathbf{b}$ & 77 \\
$\mathbf{c}$ & $\mathrm{Me}$ & $\mathrm{CH}_{2}=\mathrm{C}(\mathrm{OMe})$ & $\mathbf{a}$ & $\mathrm{Et}$ & $\mathbf{c}$ & 78 \\
$\mathbf{c}$ & $\mathrm{Me}$ & $\mathrm{CH} 2=\mathrm{C}(\mathrm{OMe})$ & $\mathbf{b}$ & $(-)-\mathrm{Men}$ & $\mathbf{d}$ & 76 \\
$\mathbf{d}$ & $n-\mathrm{Bu}$ & $t-\mathrm{CH}_{2}=\mathrm{CHCH}=\mathrm{C}(\mathrm{OMe})$ & $\mathbf{a}$ & $\mathrm{Et}$ & $\mathbf{e}$ & $63^{\mathrm{b}}$ \\
$\mathbf{e}$ & $n-\mathrm{Bu}$ & $t\left(n-\mathrm{C}_{5} \mathrm{H}_{11}\right) \mathrm{CH}=\mathrm{CH}$ & $\mathbf{a}$ & $\mathrm{Et}$ & $\mathbf{f}$ & $85^{\mathrm{c}}$ \\
$\mathbf{f}$ & $n-\mathrm{Bu}$ & $t-(\mathrm{TMS}) \mathrm{CH}=\mathrm{CH}$ & $\mathbf{a}$ & $\mathrm{Et}$ & $\mathbf{g}$ & $83^{\mathrm{d}}$ \\
\hline
\end{tabular}

${ }^{a}$ Isolated yields of analytically pure product. ${ }^{\mathrm{b}}$ A 70:30 E/Z mixture was obtained. ${ }^{\mathrm{c}}$ A 90:10 t/c mixture was obtained from 90:10 t/c 1e. ${ }^{\mathrm{d}}$ Only the trans isomer was obtained from $\mathbf{1 f}$.

\section{Experimental Section}

General Procedures. All experiments were conducted employing pre-dried glassware $(1 \mathrm{~h}$, $150{ }^{\circ} \mathrm{C}$ ) under a nitrogen atmosphere. Standard handling techniques for air-sensitive compounds were employed throughout this study. ${ }^{13} \mathrm{NMR}$ spectra were obtained on a General Electric QE300, a General Electric GN-300, a Bruker Advance DPX-300 and / or a Bruker Advance DRX500 spectrometers. ${ }^{1} \mathrm{H},{ }^{13} \mathrm{C},{ }^{11} \mathrm{~B}$ and ${ }^{29} \mathrm{Si} \mathrm{NMR}$ were recorded in $\mathrm{CDCl}_{3}$ or $\mathrm{C}_{6} \mathrm{D}_{6}$, unless otherwise used, and the chemical shifts were expressed in relative to $\mathrm{CDCl}_{3}\left(7.26\right.$ and $77.0 \mathrm{ppm}$ in ${ }^{1} \mathrm{H}$ and ${ }^{13} \mathrm{C}$ NMR, respectively ) or $\mathrm{C}_{6} \mathrm{D}_{6}$ ( 7.15 and $128.0 \mathrm{ppm}$ in ${ }^{1} \mathrm{H}$ and ${ }^{13} \mathrm{C} \mathrm{NMR}$, respectively ) as the internal standard. Multiplicity assignments and sequence in ${ }^{13} \mathrm{C}$ NMR were made with the aid of DEPT and HETCOR experiments. The ${ }^{1} \mathrm{H}-{ }^{13} \mathrm{C}$ chemical shift correlation experiment was obtained using standard sequence. ${ }^{14}$ The spectra were acquired with $4 \mathrm{~K}$ X 256 data points [ $S$ ( $\mathrm{t} 1, \mathrm{t} 2$ ) ] and a data acquisition of 16 scans $\mathrm{X} 128$ increments of $\mathrm{t}_{1}$ and zero filling in both dimensions. ${ }^{1} \mathrm{H}$ NMR assignments were carried out with the aid of ${ }^{1} \mathrm{H}-{ }^{1} \mathrm{H}$ COSY experiment. COSY-90 was collected as 1024 X 256 blocks of data, and was processed using sinusoidal multiplication in each dimension followed by symmetrization of the final data matrix. Infrared spectra were obtained on a Nicolet Magna IR-750, a Perkin-Elmer 281 or a Nicolet Series 6000 FT-IR spectrophotometers. Mass spectral data was obtained with a Hewlett-Packard 5995A GC/MS spectrometer $(70 \mathrm{eV})$. Gas chromatographic analyses were performed with a PerkinElmer 8320 capillary or a Perkin-Elmer Autosystem XL gas chromatographs using $30 \mathrm{~m} \mathrm{X} 0.25$ mm I.D. 20\% SE-30 vitreous silica open tubular columns. Optical rotation data were obtained using a Perkin-Elmer 243B Polarimeter. Elemental analyses were performed by Atlantic Microlab, Norcross, Georgia. Organostannanes were prepared by standard methods. , $^{6}$ 


\section{Palladium-catalyzed cross-coupling reactions}

Ethyl benzoate (3a). To a solution of benzyl(chloro)bis(triphenylphosphine) palladium

(II) $\left(\mathrm{BnPdCl}\left(\mathrm{PPh}_{3}\right)_{2}, 0.04 \mathrm{~g}, 0.005\right)$ and benzene $(25 \mathrm{~mL})$, were added via syringe ethyl chloroformate (2a, $0.782 \mathrm{~g}, 7 \mathrm{mmol}$ ) and 1a ( $1.46 \mathrm{~g}, 2 \mathrm{mmol})$. After refluxing for $10 \mathrm{~h}$, an additional $0.02 \mathrm{~g}$ of catalyst and $0.35 \mathrm{~g}$ of $\mathbf{2 a}$ were added to the mixture. After refluxing for $2 \mathrm{~h}$, the mixture was extracted with either a saturated solution of $\mathrm{KF} / \mathrm{NaHCO}_{3}$ or $5 \% \mathrm{NaCHO}_{3}$, dried $\left(\mathrm{Na}_{2} \mathrm{SO}_{4}\right)$, filtered over $\mathrm{Al}_{2} \mathrm{O}_{3}$, concentrated and distilled to give $0.51 \mathrm{~g}(85 \%)$ of $3 \mathbf{a}$ bp $75-7{ }^{\circ} \mathrm{C}$ (1.5 Torr). All physical and spectroscopic data were identical to those of an authentic sample of 3a. ${ }^{15 \mathrm{a}}{ }^{1} \mathrm{H} \mathrm{NMR}\left(\mathrm{CDCl}_{3} 300 \mathrm{MHz}\right) \delta 1.38(\mathrm{t}, J=7.1 \mathrm{~Hz}, 2 \mathrm{H}), 7.39-7.55(\mathrm{~m}, 3 \mathrm{H}), 8.04(\mathrm{~m}$, 2H) ppm. ${ }^{13} \mathrm{C} \mathrm{NMR}\left(\mathrm{CDCl}_{3} 75 \mathrm{MHz}\right) \delta 14.2,60.7,128.2,129.4,130.4,132.6,166.4 \mathrm{ppm}$. IR (TF) 3060, 3030, 2980, 2935, 2900, 1720, 1600, 1580, 1450, 1370, 1310, 1275, 1120, 1105, 1070, 1025, 785, $710 \mathrm{~cm}^{-1}$. GCMS m/z (relative intensity) 150 (24), 122 (34), 106 (11), 105 (100), 77 (4), 74 (11), 51 (55).

Ethyl 2-octynoate (3b). To a solution of $\mathrm{BnPdCl}\left(\mathrm{PPh}_{3}\right)_{2}(0.065 \mathrm{~g}, 0.009 \mathrm{mmol})$ in benzene $(25$ $\mathrm{mL})$ were added via syringe $\mathbf{2 a}(0.19 \mathrm{~g}, 1.77 \mathrm{mmol})$ and $\mathbf{1 b}(0.334 \mathrm{~g}, 1.3 \mathrm{mmol})$. After cooling to room temperature, pentane $(50 \mathrm{~mL})$ was added. The mixture was extracted with either a saturated solution of $\mathrm{KF} / \mathrm{NaHCO}_{3}$ or $5 \% \mathrm{NaCHO}_{3}$, dried $\left(\mathrm{Na}_{2} \mathrm{SO}_{4}\right)$, filtered over $\mathrm{Al}_{2} \mathrm{O}_{3}$, concentrated and distilled to give $0.20 \mathrm{~g}(77 \%)$ of $3 \mathbf{b}$ bp $127{ }^{\circ} \mathrm{C}$, Kugelrohr (8.5 Torr). All physical and spectroscopic data were identical to those of an authentic sample of $3 \mathrm{a}^{15 \mathrm{~b}}{ }^{1} \mathrm{H}$ NMR $\left(\mathrm{CDCl}_{3}\right.$ $300 \mathrm{MHz}) \delta 0.81(\mathrm{t}, J=6.8 \mathrm{~Hz}, 3 \mathrm{H}), 1.18-1.32(\mathrm{~m}, 9 \mathrm{H}), 1.47$ (qt, $J=7.0 \mathrm{~Hz}, 2 \mathrm{H}), 2.23(\mathrm{t}, J=$ $7.0 \mathrm{~Hz}, 2 \mathrm{H}), 4.12(\mathrm{q}, J=7.0 \mathrm{~Hz}, 2 \mathrm{H}) \mathrm{ppm} .{ }^{13} \mathrm{C} \mathrm{NMR}\left(\mathrm{CDCl}_{3} 75 \mathrm{MHz}\right) \delta 13.6,13.8,18.3,21.9$, 27.0, 30.7, 61.4, 73.0, 89.0, 153.5 ppm. IR (TF) 2960, 2945, 2880, 2230, 1710, 1470, 1360, $1250,1070,1050,880,750 \mathrm{~cm}^{-1}$. GCMS m/z (relative intensity) 168 (4), 164 (17), 139 (15), 125 (17), 123 (77), 111 (24), 97 (20), 95 (79), 94 (27), 93 (46), 91 (23), 84 (28), 81 (27), 80 (16), 79 (58), 77 (23), 88 (16), 87 (90), 86 (100), 85 (24), 55 (43), 53 (35), 51 (22).

Ethyl 2-methoxypropenoate (3c). As for 3b, using $\mathrm{BnPdCl}\left(\mathrm{PPh}_{3}\right)_{2}(0.35 \mathrm{~g}, 0.05 \mathrm{mmol})$, benzene $(200 \mathrm{~mL}), 2 \mathrm{a}(3.92 \mathrm{~g}, 35 \mathrm{mmol})$ and $1 \mathrm{c}(7.33 \mathrm{~g}, 35.0 \mathrm{mmol})$ after $16 \mathrm{~h}$ at reflux, $3.56 \mathrm{~g}$ (78 \%) of 3c was obtained, bp 108-110 ${ }^{\circ} \mathrm{C}$ (85 Torr). All physical and spectroscopic data were identical to reported values. ${ }^{16}{ }^{1} \mathrm{HMR}\left(\mathrm{CDCl}_{3} 300 \mathrm{MHz}\right) \delta 1.32(\mathrm{t}, J=7.0 \mathrm{~Hz}, 3 \mathrm{H}), 3.65(\mathrm{~s}, 3 \mathrm{H})$, $4.27(\mathrm{q}, J=7.0 \mathrm{~Hz}, 2 \mathrm{H}), 4.60(\mathrm{~d}, J=2.7 \mathrm{~Hz}, 1 \mathrm{H}), 5.44\left(\mathrm{~d}, J=2.7 \mathrm{~Hz},{ }^{1} \mathrm{H}\right) \mathrm{ppm} .{ }^{13} \mathrm{C}$ NMR (CDCl3 $75 \mathrm{MHz}) \delta 14.0,55.5,61.3,93.1,152.3,162.9$ ppm. IR (TF) 2990, 2920, 2860, 1745, $1630,1450,1360,1325,1205,1050,1020,850,790 \mathrm{~cm}^{-1}$. GCMS m/z (relative intensity) 130 $\left(\mathrm{M}^{+}, 46\right), 101$ (34), 5 (31), 84 (31), 73 (48), 58 (34), 57 (100), 56 (28), 55 (57), 53 (7).

(-)-Menthyl 2-methoxypropenoate (3d). As for 3b, using $\mathrm{BnPdCl}\left(\mathrm{PPh}_{3}\right)_{2}(0.33 \mathrm{~g}, 0.045 \mathrm{mmol})$, benzene $(200 \mathrm{~mL}),(-)$-menthyl chloroformate $(2 \mathbf{b}, 6.56 \mathrm{~g}, 30 \mathrm{mmol})$ and $\mathbf{1 c}(6.62 \mathrm{~g}, 30.0 \mathrm{mmol})$ afforded after refluxing for $16 \mathrm{~h}, 5.16 \mathrm{~g}(76 \%)$ of $3 \mathrm{~d}$ bp $132{ }^{\circ} \mathrm{C}(0.82$ Torr $)[\alpha]_{\mathrm{D}}{ }^{7}=-84^{\circ}(\mathrm{c}=$ 0.112, $\left.\mathrm{CHCl}_{3}\right)$. Anal. Calcd. for $\mathrm{C}_{14} \mathrm{H}_{24} \mathrm{O}_{3}: \mathrm{C}, 69.95, \mathrm{H}, 10.07$. Found: $\mathrm{C}, 70.01, \mathrm{H}, 10.03 .{ }^{1} \mathrm{H}$ NMR $\left(\mathrm{CDCl}_{3} 300 \mathrm{MHz}\right) \delta 0.72(\mathrm{~d}, J=7.0 \mathrm{~Hz}, 3 \mathrm{H}), 0.84-0.87(\mathrm{~m}, 6 \mathrm{H}), 0.89-1.07(\mathrm{~m}, 4 \mathrm{H}), 1.39-$ $1.52(\mathrm{~m}, 1 \mathrm{H}), 1.62-1.66(\mathrm{~m}, 4 \mathrm{H}), 1.79-1.89(\mathrm{~m}, 1 \mathrm{H}), 1.96-2.02(\mathrm{~m}, 2 \mathrm{H}), 3.60(\mathrm{~s}, 3 \mathrm{H}), 4.56(\mathrm{~d}, J=$ $2.6 \mathrm{~Hz}, 1 \mathrm{H}), 4.77(\mathrm{dt}, J=10.9,4.4 \mathrm{~Hz}, 1 \mathrm{H}), 5.27(\mathrm{~d}, J=2.6 \mathrm{~Hz}, 1 \mathrm{H}) \mathrm{ppm} .{ }^{13} \mathrm{C} \mathrm{NMR}\left(\mathrm{CDCl}_{3}\right.$ 
$75 \mathrm{MHz}) \delta 16.1,20.3,21.7,23.2,26.0,31.1,33.9,40.3,46.7,74.9,92.6,152.1,162.2 \mathrm{ppm} . \mathrm{IR}$ (TF) 3010, 2950, 2870, 1760, 1650, 1460, 1200, 1180, 1030, 990, 840, $750 \mathrm{~cm}^{-1}$. GCMS m/z (relative intensity) 138 (36), 104(17), 95 (61), 83 (52), 81 (52), 69 (29), 67 (21), 57 (100), 55 (58).

Ethyl 2-methoxy-(2E,4)-pentadienoate (3e). As for 3b, using $\mathrm{BnPdCl}\left(\mathrm{PPh}_{3}\right)_{2}(0.65 \mathrm{~g}$, $0.09 \mathrm{mmol})$, benzene $(100 \mathrm{~mL}), 2 \mathrm{a}(0.45 \mathrm{~g}, 4.2 \mathrm{mmol})$ and 1d ((Z)-1-tributylstannyl-1-methoxy1,3-butadiene, $1.35 \mathrm{~g}, 3.6 \mathrm{mmol})$ afforded $0.35 \mathrm{~g}(61 \%)$, of 3e as a 70:30 E/Z mixture bp 115$118^{\circ} \mathrm{C}\left(2\right.$ Torr). ${ }^{18}{ }^{1} \mathrm{H} \mathrm{NMR}\left(\mathrm{CDCl}_{3} 300 \mathrm{MHz}\right)$ (major isomer) $\delta 1.34(\mathrm{t}, J=7.0 \mathrm{~Hz}, 3 \mathrm{H}), 3.66(\mathrm{~s}$, $3 \mathrm{H}), 4.30(\mathrm{q}, J=7.0 \mathrm{~Hz}, 2 \mathrm{H}), 5.85(\mathrm{~d}, J=10.9 \mathrm{~Hz}, 1 \mathrm{H}), 5.14-5.32(\mathrm{~m}, 2 \mathrm{H}), 7.18-7.31(\mathrm{~m}$, $1 \mathrm{H}) \mathrm{ppm}$, (minor isomer) $1.29(\mathrm{t}, J=7.0 \mathrm{~Hz}, 3 \mathrm{H}), 3.71(\mathrm{~s}, 3 \mathrm{H}), 4.28(\mathrm{q}, J=7.0 \mathrm{~Hz}, 2 \mathrm{H}), 5.33-$ $5.51(\mathrm{~m}, 2 \mathrm{H}), 6.65-6.71(\mathrm{~m}, 1 \mathrm{H}), 6.75-6.77(\mathrm{~m}, 1 \mathrm{H}) \mathrm{ppm} .{ }^{13} \mathrm{C} \mathrm{NMR}\left(\mathrm{CDCl}_{3} 75 \mathrm{MHz}\right)$ (major isomer) $\delta 14.2,55.6,61.2,113.7,119.1,131.7,146.0,163.0 \mathrm{ppm}$, (minor isomer) 14.1, 60.4, 60.9, 122.5, 125.5, 129.7, 145.6 ppm. IR (TF) 3095, 2980, 2880, 1720, 1630, 1590, 1450, 1370, $1345,1240,1180,1140,1100,1030,1100,1000,920,780,730 \mathrm{~cm}^{-1}$. GCMS m/z (relative intensity) 156 (72), 128 (34), 127 (40), 117 (70), 98 (33), 83 (34), 78 (280, 69(43), 68 (100).

Ethyl trans-2-octenoate (3f). As for $\mathbf{3 b}$, using $\mathrm{BnPdCl}\left(\mathrm{PPh}_{3}\right)_{2}(0.08 \mathrm{~g}, 0.01 \mathrm{mmol})$, benzene $(25 \mathrm{~mL}), 2 \mathrm{a}(1.09 \mathrm{~g}, 10 \mathrm{mmol})$ and $1 \mathbf{e}(90: 10 \mathrm{t} / \mathrm{c}$-1-heptenyltri-n-butylstannane, $3.10 \mathrm{~g}, 8 \mathrm{mmol})$. After $6 \mathrm{~h}, 0.03 \mathrm{~g}$ of catalyst and $1.5 \mathrm{~g}$ of $2 \mathrm{a}$ were added. After refluxing for $3 \mathrm{~h}, 1.16 \mathrm{~g}(85 \%)$ of 3f were obtained as a trans-cis mixture $(90: 10)$ bp $70-72{ }^{\circ} \mathrm{C}$ (1.5 Torr). All physical and spectroscopic data were identical to those of an authentic sample of $3 a^{15 b}{ }^{1} \mathrm{H}$ NMR $\left(\mathrm{CDCl}_{3}\right.$ $300 \mathrm{MHz}$ ) (major isomer) $\delta 0.88(\mathrm{~m}, 6 \mathrm{H}), 1.28(\mathrm{~m}, 4 \mathrm{H}), 1.44(\mathrm{~m}, 2 \mathrm{H}), 2.18(\mathrm{~m}, 2 \mathrm{H}), 4.15(\mathrm{q}, J=$ $6.6 \mathrm{~Hz}, 2 \mathrm{H}), 5.79(\mathrm{~d}, J=15.4 \mathrm{~Hz}, 1 \mathrm{H}), 6.94(\mathrm{dt}, J=15.4,6.6 \mathrm{~Hz}, 1 \mathrm{H}) \mathrm{ppm} .{ }^{13} \mathrm{C} \mathrm{NMR}\left(\mathrm{CDCl}_{3} 75\right.$ $\mathrm{MHz}$ ) (major isomer) $\delta 13.7,14.0,22.2,27.6,31.2,32.0,59.9121 .1,149.2,165.5 \mathrm{ppm}$, (minor isomer) $\delta 13.8,14.0,22.0,27.7,31.5,59.5,119.4,150.3,166.3$ ppm. IR (TF) 2950, 2900, 2850, $1730,1655,1460,1365,1305,1260,1200,1170,1125,1040,985,860 \mathrm{~cm}^{-1}$. GCMS m/z (relative intensity) 170 (2), 125 (42), 124 (20), 101 (31), 99 (31), 96 (23), 88 (17), 82 (22), 73 (44), 68 (25), 55 (100).

Ethyl trans-3-trimethylsilyl-2-propenoate (3g). As for 3f, using $\mathrm{BnPdCl}\left(\mathrm{PPh}_{3}\right)_{2}(0.08 \mathrm{~g}$, $0.01 \mathrm{mmol})$, benzene $(25 \mathrm{~mL}), \mathbf{2 a}(1.04 \mathrm{~g}, 9.6 \mathrm{mmol})$ and $\mathbf{1 f}(3.73 \mathrm{~g}, 9.6 \mathrm{mmol})$. After refluxing for $6 \mathrm{~h}, 1.14 \mathrm{~g}(83 \%)$ of $3 \mathrm{~g}$ were obtained, bp $90-92{ }^{\circ} \mathrm{C}$ (1.5 Torr). All physical and spectroscopic data were identical to reported values. ${ }^{17}{ }^{1} \mathrm{H} \mathrm{NMR}\left(\mathrm{CDCl}_{3} 300 \mathrm{MHz}\right) \delta 0.12(\mathrm{~s}, 9 \mathrm{H})$, 1.30 (t, $J=7.1 \mathrm{~Hz}, 3 \mathrm{H}), 4.20$ (q, $J=7.1 \mathrm{~Hz}, 2 \mathrm{H}), 6.23$ (d, $J=19.0 \mathrm{~Hz}, 1 \mathrm{H}), 7.25$ (d, $J=19.0 \mathrm{~Hz}$, $1 \mathrm{H}) \mathrm{ppm} .{ }^{13} \mathrm{C} \mathrm{NMR}\left(\mathrm{CDCl}_{3} 75 \mathrm{MHz}\right) \delta-2.3,13.9,60.0,133.9,148.7,165.2 \mathrm{ppm}$. IR (TF) 3010, 2980, 2880, 2670, 1720, 1600, 1430, 1360, 1300, 1250, 1230, 1160, 1030, 990, 840, 750, $685 \mathrm{~cm}^{-1}$. GCMS m/z (relative intensity) 171 (0.9), 158 (12), 157 (100), 121 (31), 103 (25), 99 (12), $\quad 83 \quad(35), \quad 75 \quad(66), \quad 73 \quad(63), \quad 59 \quad(20), \quad 58 \quad(25), \quad 53 \quad$ (17). 


\section{Acknowledgements}

The support of NIH-MBRS (SO6-GM08102), DOE (DE-FCO2-91ER75674) and NSF (CHE9817550) is gratefully acknowledged as is the assistance of Dr. G. P. Roth.

\section{References and Notes}

1. This work is dedicated to Professor Alfred Hassner, a great scientist, teacher and mentor on the occasion of his $70^{\text {th }}$ birthday.

2. Graduate student supported by the NIH-MBRS program (SO6-GM08102).

3. Undergraduate student supported by the NSF-RCMS program (HRD-9011964).

4. Summer undergraduate internship through the NIH-MBRS program (SO6-GM08216-13, UPR-Humacao).

5. (a) Stille, J. K. Angew. Chem., Int. Ed. 1986, 25, 508. (b) Mitchell, T. N. Synthesis 1992, 803. (c) Farina, V.; Roth, G. P. In Advances in Metal-Organic Chemistry; Liebeskind, L. S. Ed.; Jai Press: Greenwich, 1995; Vol. 5. (d) Farina, V.; Krishnamurthy, V.; Scott, W. J. Org. React. 1997, 50, 1.

6. (a) Soderquist, J. A.; Lopez, C. Tetrahedron Lett. 1991, 32, 6305. (b) Soderquist, J. A.; Hassner, A. J. Am. Chem. Soc. 1980, 102, 1577. (c) Soderquist, J. A.; Hsu, G. J.-H. Organometallics 1982, 1, 830.

7. (a) Farina, V.; Kapadia, S.; Krishnan, B.; Wang, C.; Liebeskind, L. S. J. Org. Chem. 1994, 59, 5905. (b) Piers, E.; Wong, T. J. Org. Chem. 1993, 58, 3609. (c) Ellman, J. A.; Plunkett, M. J. J. Am. Chem. Soc. 1995, 117, 3306. (d) Roth, G. P.; Farina, V.; Pena-Cabrera, E. Tetrahedron Lett. 1995, 36, 2191. (e) Casado, A. L.; Espinet, P. J. Am. Chem. Soc. 1998, 120, 8978. (f) Maleczka Jr., R. E.; Terstiege, I. J.Org. Chem. 1998, 63, 9622. (d) Han, X.; Stoltz, B. M.; Corey, E. J. J. Am. Chem. Soc. 1999, 121, 7600.

8. (a) Nicolaou, K. C.; Chakraborty, T. K.; Piscopio, A. D.; Minowa, N.; Bertinato, P. J. Am. Chem. Soc. 1993, 115, 4419. (b) Pattenden, G.; Thom, S. M. Synlett 1993, 215 (c) Newhouse, B. J.; Meyers, A. I.; Sirisoma, N. S.; Braun, M. P.; Johnson, C. R. Synlett 1993, 573. (d) Shair, M. D.; Yoon, T.; Danishefski, S. J. J. Org. Chem. 1994, 59, 3755. (e) Cummins, C. H. Tetrahedron Lett. 1994, 35, 857. (f) Badone, D.; Cardamone, R.; Guzzi, U. Tetrahedron Lett. 1994, 35, 5477. (g) Liebeskind, L.S.; Stone, G. B.; Zhang, S. J. Org. Chem. 1994, 59, 7917. (h) Tsuji, Y.; Obora, Y.; Kawamura, T. J. Org. Chem. 1995, 60, 4647. (i) Wang, J.; Scott, I. Tetrahedron Lett. 1995, 36, 7043. (j) Barsanti, P. A.; Armstrong, A. Synlett. 1995, 903. (k) Hitchcock, S. A.; Mayhugh, D. R.; Gregory, G. S. Tetrahedron Lett. 1995, 36, 9085. (1) Bao, Z.; Chan, W. K.; Yu, L. J. Am. Chem. Soc. 1995, 117, 12426. (m) Nakamura, H.; Takeuchi, D.; Murai, A. Synlett. 1995, 1227. (n) Entwistle, D. A.; Jordan, S. I.; Pattenden, G. Perkin Trans. 1 1994, 1697. (o) Bunz, U. H. F. Chem. Ber. 1996, 1311. (p) Kennedy, G.; Perboni, A. D. Tetrahedron Lett. 1996, 37, 7611. (q) Nicolaou, K. 
C.; Postema, M. H. D.; Paquette, L. A. Chemtracts 1997, 10, 14. (r) Kollar, L.; SkodaFoldes, R.; Tuba, Z. J. Org. Chem. 1997, 62, 1326. (s) Godt, A. J. Org. Chem. 1997, 62, 7471. (t) Jeannert, V.; Meerpoel, L.; Vogel, P. Tetrahedron Lett. 1997, 38, 543. (u) Paterson, I.; Man, J. Tetrahedron Lett. 1997, 38, 695. (v) Dondoni, A.; Ghiglione, C.; Scoponi, M. J. Org. Chem. 1998, 63, 9395. (w) Quayle, P.; Wang, J.; Urch, C. J. Tetrahedron Lett. 1998, 39, 485. (y) Dykstra, K. D.; DiNinno, F. Tetrahedron Lett. 1998, 39, 1865. (z) Smith III, A. B.; Ott, G. R. J. Am. Chem. Soc. 1998, 120, 3935. (aa) Entwistle, D. A.; Jordan, S. I.; Pattenden, G. Synthesis 1998, 603. (ab) Chamoin, S.; Houldsworth, S.; Snieckus, V. Tetrahedron Lett. 1998, 39, 4175. (ac) Duchene, A.; Parrian, J.-L. Prie, G. Synlett. 1998, 839. (ad) Hucke, A.; Cava, M. P. J. Org. Chem. 1998, 63, 7413. (ae) Olivera, R.; Pascual, S.; Dominguez, E. Tetrahedron Lett. 1998, 39, 7155. (af) Betzer, J.-F.; Lallemand, J.-Y.; Pancrazi, A. Synthesis 1998, 522. (ag) Kang, S,-K.; Lee, Y.-T.; Lee, S,-H. Tetrahedron Lett. 1999, 40, 3573. (ah) Mowery, M. E.; DeShong, P. J. Org. Chem. 1999, 64, 1684. (ai) Buynak, J. D.; Doppalapudi, V. R.; Frotan, M.; Kumar, R. Tetrahedron Lett. 1999, 40, 1281. (aj) Tuozzi, A.; Lo Sterzo, C.; Bocelli, G. Tetrahedron 1999, 55, 461. (ak) Benhida, R.; Lecubin, F.; Quintero, L. Tetrahedron Lett. 1999, 40, 5701. (al) Aranyos, V.; Castano, M.; Grennberg, H. Acta Chemica Scandinavia. 1999, 53, 714. (am) Kuribayashi, T.; Gohya, S.; Satoh, S. J. Carbohydrate Chem. 1999, 18, 383. (an) Jung, Y.-S.; Joe, B.-Y.; Park, N.-S. Bull. Korean Chem. Soc. 2000, 21, 463. (ao) J. Org. Chem. 2000, 65, 5917. (ap) Lee, C. Y.; Hanson, R. N. Tetrahedron 2000, 56, 1623. (aq) Richecoeur, A. M. E.; Sweeney, J. B. Tetrahedron 2000, 56, 389. (ar) Paulon, A.; Cossu, S.; Zonta, C. Chem. Commun. 2000, 1839. (as) Liu, C.-M.; Luo, S.-J.; Ma, Y.-X. Synth. Commun. 2000, 2281.

9. Soderquist, J. A.; Leong, W. W-H. Tetrahedron Lett. 1983, 24, 2361.

10. (a) Balas, L.; Jousseaume, B.; Shin, H.-A.; Verlhac, J.-B.; Walian, F. Organometallics 1991, 10, 366. (b) Jousseaume, B.; Kwon, H.-A.; Verlhac, J.-B.; Denat, F.; Dubac, J. Synlett 1993, 117. (c) Adlington, R. M.; Baldwin, J. E.; Russell, T. J. Chem. Soc. Perkin Trans.1 1994, 1697.

11. (a) March, J. Advanced Organic Chemistry; $4^{\text {th }}$ Edn; Wiley: New York, 1992; pp 618-19 and ref. cited therein. (b) Zweifel, G.; Miller, J. A. Org. React. 1984, 32, 375.

12. Stille, J. K.; Groh, B. L. J. Am. Chem. Soc. 1987, 109, 813.

13. Brown, H. C.; Midland, M.M.; Levy, L. V.; Kramer, G. W. Organic Syntheses via Boranes; Wiley-Interscience: New York, 1975.

14. (a) Maudsley, A. A.; Ernst, R. R. Chem Phys. Lett. 1977, 50, 368. (b) Bodenhausen; Morris, G. A. J. Chem. Soc., Chem. Commun 1978, 320. (c) Freeman, R.; Morris, G. A. J. Chem. Soc., Chem. Commun 1978, 684.

15. (a) Available from Aldrich Chemical Co. (b) Available from Lancaster Synthesis.

16. Ochiai, H.; Tamaru, Y.; Tsubaki, K.; Yoshidu, Z. J. Org. Chem. 1987, 52, 4420.

17. Yi, C.; He, Z.; Lee, D. Organometallics 2000, 19, 2036.

18. Our attempts to obtain an acceptable elemental analysis for this unstable isomeric mixture were unsuccessful. Our NMR data is consistent with those reported for the corresponding 
isomeric methyl esters (Stevenart-De Mesmaeker, N.; Merenyi, R.; Viehe, H. G. Tetrahedron Lett. 1987, 28, 2591). This problem is evidently common for such dienoates with the reported isomeric methyl esters also being analyzed only spectroscopically. 